\title{
Regression Models for Texture Image Analysis
}

\author{
Anatoliy Plastinin \\ Samara State Aerospace University, \\ Molodogvardeiskaya st 151, Samara, 443001 Russia \\ anatoliy.plastinin@gmail.com
}

\begin{abstract}
The article describes universal model for creating algorithms for calculating textural image features. The proposed models are used for images that are realizations of Markov Random Field. Experimental classification results are shown for different images sets.
\end{abstract}

Keywords: Textutre Image Analysis, Textutre Image Recognition, Regression models, Markov Random Fields.

\section{Introduction}

Many natural micro and macro images are combinations of textural primitives, e.g. aerial photographs of the earth surface, images of material nanostructures, biomedical images. That's why in many applied problems of the image processing (like, segmentation, coding, simulation, etc.) texture processing methods can be used.

Two main approaches to the texture analysis can be noted: structural and stochastic [1. In structural approach textures are considered as a combination of repeating primitive elements, which relative position is defined by specific rules. In stochastic approach, the texture is defined as a realization of a $2 \mathrm{D}$ random process, and specified by a set of random statistics. In our research work we used statistical approach.

It should be noted, that common texture analysis methods, like co-occurrence matrix [2, spectral descriptors, Tamura features 3], etc., give common features for all types of images and ignore problem peculiarities. However, in practice it becomes necessary to calculate features for classification of limited texture sets. Also many methods deal with gray-scale images only i.e. these methods can't be applied to analysis of some classes of texture images. Methods based on Markov random field are successfully used in many applied problems. This methods proved to be efficient in image segmentation problems, image synthesis, etc. 4567 . Thus, the goal of our investigation is to provide general framework for estimation of color-texture features based on domain specific information.

\section{Problem of Texture Image Recognition}

Let $\mathfrak{I}$ is a set of all possible images, that consists of $K$ classes $C_{1}, \ldots, C_{K}$, i.e. $\bigcup_{k=0}^{K} C_{k}=\mathfrak{I}$ and $C_{i} \cap C_{j}=\varnothing, i \neq j$, here $C_{0}$ - set of objects that are not included in any class. Original class separation is unknown. 
Suppose we are given a finite set of images $\mathcal{I}=\left\{I_{1}, \ldots, I_{N}\right\} \subset \mathfrak{I}$, the class label is specified for each image from this set, i.e. following map is defined $u$ : $\mathcal{I} \rightarrow \mathfrak{L}$, where $\mathfrak{L}=\{1, \ldots, K\}$ is a set of class labels. A training set is defined as a set of image-class pairs: $T=\mathcal{I} \times \mathfrak{L}$.

The goal is to design a method that allows to identify class label based on image $I$. To use pattern recognition theory $7[8$, one should associate features vector $f \in \mathfrak{F}=\mathbb{R}^{n}$ with each image, thus following map should be defined: $F: \mathfrak{I} \rightarrow \mathfrak{F}$, where $\mathfrak{F}$ is a feature space. Hence, here exists an unknown dependence $\hat{u}: \mathfrak{F} \rightarrow \mathfrak{L}$, values are observed on finite training set $\hat{T}=\left\{\left(f_{i}, l_{i}\right)\right\}_{i=1}^{N}$. One should define a map $\tilde{u}: \mathfrak{F} \rightarrow \mathfrak{L}$, that is an approximation of unknown target dependency on both elements of training set and whole set $\mathfrak{F}$.

All existing feature calculation methods are defined for a wide range of problems, i.e. it doesn't fully utilize domain specific information from training set.

To exploit domain specific information we should define parametric feature calculation method $F_{a}$, where $a$ is a set of method parameters. Thus, specific method parameters $a=P(\mathcal{I})$ are calculated based on training set $\mathcal{I}$ at a training step, and feature vector is estimated for each specific image using calculated parameters vector $f=F_{a}(I)$. The general classification scheme is shown in figure 1 .

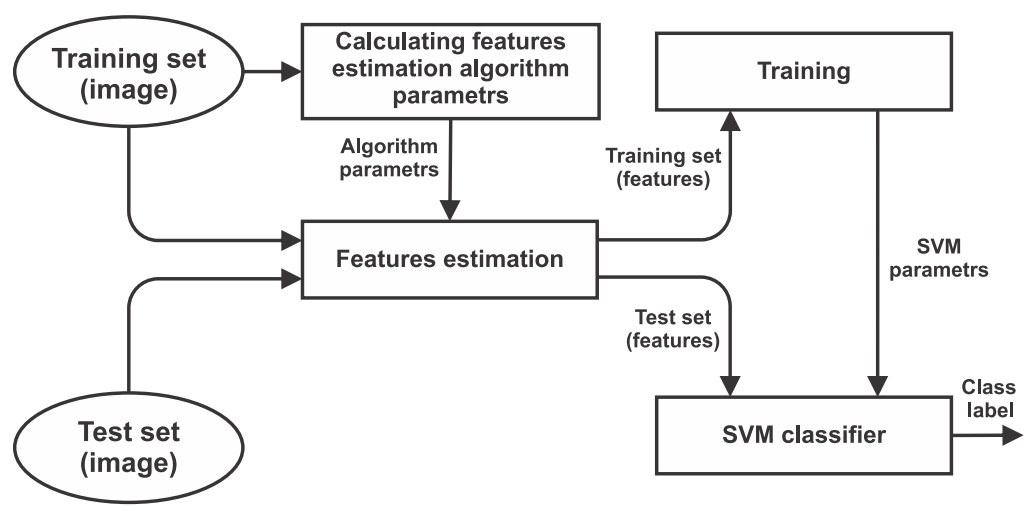

Fig. 1. Classification scheme

On first step the method's vector parameters $a=P(\mathcal{I})$ are calculated based on training set $\mathcal{I}$. Then resulted parameters used for estimation of features are used on next classifier training step.

\section{Image Model}

To design features estimation algorithm in case of texture images analysis, one can use Markov Random Field (MRF) model [7, it's effectively used in many texture analysis problems. 
Let's define image as map:

$$
I: L_{x} \times L_{y} \rightarrow G
$$

where $L_{x}=\left\{1, \ldots, K_{x}\right\}$ and $L_{y}=\left\{1, \ldots, K_{y}\right\}$ are spatial coordinates, and $G$ is a set of colors; e.g. for gray-scale image $G=[0 ; 1]$, and for colored image (RGB color space) $G=[0 ; 1]^{3}$.

Let the image be a realization of any Markov random field [7]. Following notation will be used below:

$-\mathcal{N}_{p}$ - neighborhood of a pixel $p$

$-\mathcal{N}_{p}^{k}-k$-th neighborhood element;

$-I_{\mathcal{N}_{p}}=\left(I_{\mathcal{N}_{p}^{1}}, \ldots, I_{\mathcal{N}_{p}^{K}}\right)$ - vector of pixel values in the neighborhood of current pixel.

\section{Features Design Method}

Since the value of central pixel $I_{p}$ depends on values of pixels in the neighborhood, we can consider approximation function - the function where the pixels from the neighborhood are arguments and the value of function is an estimate of central pixel value. This function can be defined as:

$$
\hat{I_{p}}=f\left(I_{\mathcal{N}_{p}}\right)=\underset{x}{\arg \max } \mathrm{P}\left(x \mid I_{\mathcal{N}_{p}}\right)
$$

or using regression equation:

$$
\hat{I_{p}}=f\left(I_{\mathcal{N}_{p}}\right)=\mathrm{E}\left[x \mid I_{\mathcal{N}_{p}}\right]
$$

Let's consider solving regression problem. $D=\left\{\left(x_{i}, y_{i}\right)\right\}=\left\{\left(I_{\mathcal{N}_{p}}, I_{p}\right) \mid p \in L_{x} \times\right.$ $\left.L_{y}\right\} \subset \mathbb{R}^{K} \times \mathbb{R}$ - training set of regression problem.

We use kernel ridge regression method [9]. According to representer theorem [8] we're going to find solution in form:

$$
\hat{f}(x)=\sum_{i=1}^{l} \alpha_{i} k\left(x, x_{i}\right),
$$

where $k$ is positive defined kernel 8 .

The cost function, which needs to be minimized for ridge regression is:

$$
\sum_{i=1}^{l}\left(y_{i}-\alpha_{i} k\left(x, x_{i}\right)\right)^{2}+\lambda \sum_{i=1}^{l} \alpha_{i}^{2} .
$$

The ridge model coefficients are given by: $\alpha=(K+\lambda I)^{-1} y$, where $K_{i, j}=$ $k\left(x_{i}, x_{j}\right)$. We use Gaussian kernel in our study:

$$
k\left(x, x^{\prime}\right)=\exp \left(-\gamma\left\|x-x^{\prime}\right\|\right) .
$$


Hence, each image is associated with its own set of parameters $\alpha$. Overall, the parameters are similar for images from the same class.

To build features separating several classes, we should use regression function based on all images from training set. But it leads to the next issue - increasing of regression problem training set size. To avoid increasing of the set size, only a subset of training set for image can be used, it's possible because a lot of vectors in the training set are similar. Hence, only linear independent (in RKHS for kernel $k$ ) vectors can be used from the training set.

\section{$5 \quad$ Selecting of Linearly Independent Subset}

Consider we need to select $M$ linear independent vectors from set $\left\{x_{i}\right\}_{i=1}^{N}$. Let $\phi(x)$ be a fixed finite dimensional mapping from domain space to Reproducing Kernel Hilbert Space with kernel $k$, such that $k(x, y)=\langle\phi(x), \phi(y)\rangle[9$.

Let's use following iterative procedure [10. On each step $t$, subset of vectors $D_{t-1}=\left\{\tilde{x}_{j}\right\}_{i=1}^{m_{t-1}}$ have been collected, after $t-1$ vectors were observed, where $\left\{\phi\left(\tilde{x}_{j}\right)\right\}_{i=1}^{m_{t-1}}$ are approximately linearly independent vectors by construction. We test if $\phi\left(x_{t}\right)$ is approximately linearly dependent on subset $D_{t-1}$, if not we add it to $D_{t-1}$, i.e. $D_{t}=D_{t-1} \cup x_{t}$. Hence, all vectors $x_{1}, \ldots, x_{t}$ can be approximated as linear combination of vectors $D_{t} . x_{t}$ is approximately linearly dependent on set $D_{t-1}$, it means that it satisfies following condition:

$$
\min \left\|\sum_{j=1}^{m_{t-1}} a_{j} \phi\left(\tilde{x}_{j}\right)-\phi\left(x_{t}\right)\right\|^{2}<\delta,
$$

where $\delta$ is accuracy parameter.

To sum it all up, the set of method parameters $a$ for each particular problem is a set of linear independent neighborhood vectors, generated from all training set images. Thus, feature vector is regression coefficients of approximation function.

\section{Experiments with Test Images}

Classification experiments on 2 sets of test images were performed, each set of images consists of 2 classes (see Fig. 2 and 3). N indicates the number of linearly independent vectors used, and $\mathrm{S}$ indicates the radius of non-causal neigborhood (e. g. S1; N5 means that $3 \times 3$ non-causal neigborhood used and number of linearly independent vectors was 5 ).

Non-linear SVM classifier [811] is used for experiments. Each class of images is split into training set of 10 images and validation set of 100 images. Training set is used to estimate parameters of feature calculation algorithm, that are used to calculate features for images in test set. Then these features are used for training of the SVM classifier. Accuracy of classification was investigated for images distorted by additive and impulse noise, using the decision function obtained on previous step. The results are shown in Fig. 4 and 5. 

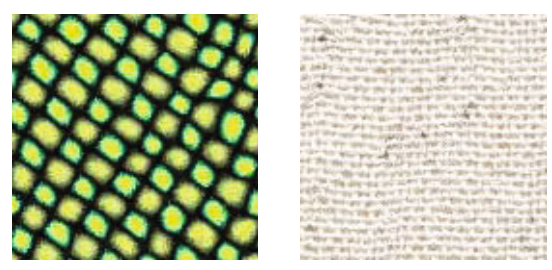

Fig. 2. Sample images from 1st set
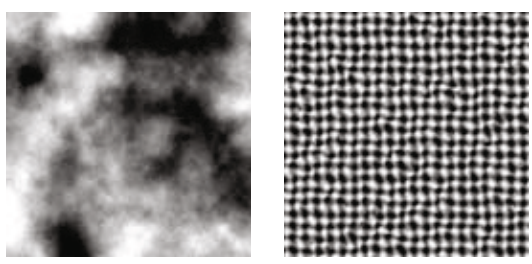

Fig. 3. Sample images from 2 nd set

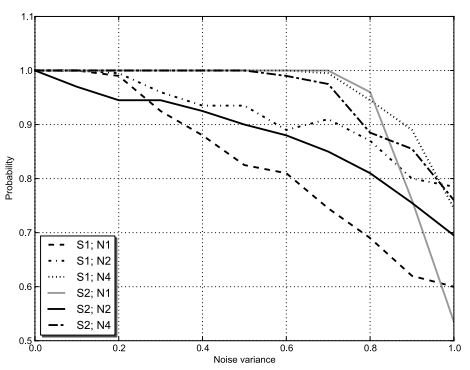

a

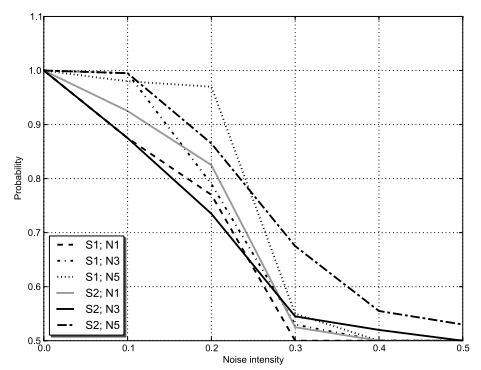

b

Fig. 4. Effect of white and impulse noise on 1st image set: a — white noise, b impulse noise

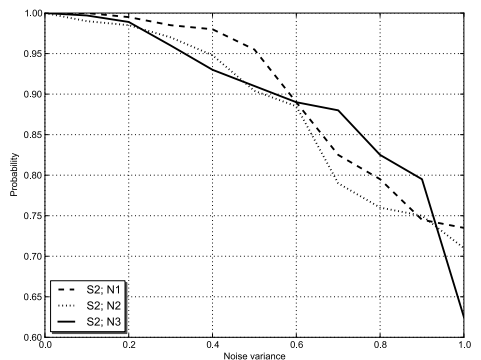

a

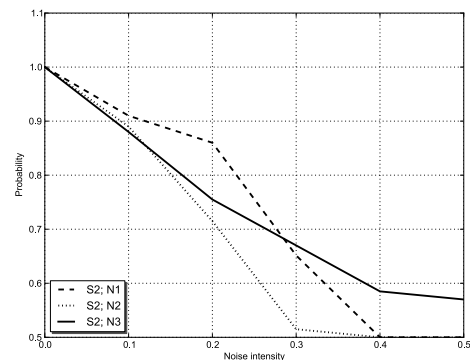

$\mathrm{b}$

Fig. 5. Effect of white and impulse noise on 2nd image set: a - white noise, b impulse noise 


\section{Conclusion}

In our research work the general framework for estimating textural features, which utilizes domain specific information from training set, is described. Proposed method is used for formalization of features estimation algorithm based on approximation of pixels dependency function, for images with markov random field model.

The method has following advantages: domain specific features increase classification quality (in terms of correct classification probability); features are stable to additive and impulse noise.

\section{References}

1. Haralick, R.M.: Statistical and structural approaches to texture. Proceedings of the IEEE 67(5), 786-804 (1979)

2. Haralick, R.M., Shanmugam, K., Dinstein, I.: Textural Features for Image Classification. IEEE Transactions on Systems, Man, and Cybernetics SMC-3(6), 610-621 (1973)

3. Tamura, H., Mori, S., Yamawaki, T.: Textural Features Corresponding to Visual Perception. IEEE Transaction on Systems, Man, and Cybernetcs SMC-8(6), 460$472(1978)$

4. Winkler, G.: Image Analysis, Random Fields and Dynamic Monte Carlo Methods, p. 324. Springer, Heidelberg (1995)

5. Paget, R.: Nonparametric Markov Random Field Models for Natural Textures Images, Ph.D. thesis, University of Queensland, St Lucia, QLD Australia (December 1999)

6. Kaulgud, N., Desai, U.B.: Efficient color image restoration using Markov random field. In: TENCON 1998. 1998 IEEE Region 10 International Conference on Global Connectivity in Energy, Computer, Communication and Control, vol. 1, pp. 41-44 (1998)

7. Li, S.Z.: Markov Random Field Modeling in Image Analysis, 3rd edn. Springer, Heidelberg (2009)

8. Scholkopf, B., Smola, A.J.: Learning with Kernels Support Vector Machines, Regularization, Optimization, and Beyond. MIT Press, Cambridge (2001)

9. Hastie, T., Tibshirani, R., Friedman, J.: The Elements of Statistical Learning. Data Mining, Inference, and Prediction, 2nd edn. Springer, Heidelberg (2009)

10. Engel, Y., Mannor, S., Meir, R.: The Kernel Recursive Least-Squares Algorithm. IEEE Transactions on Signal Processing 52(8) (2004)

11. Vapnik, V.: Statistical Learning Theory. Wiley, Chichester (1998) 